\title{
Conference Paper \\ Molecular Determinants for the Self-Assembly of Elastin Peptides
}

\author{
Brigida Bochicchio, Maria Rosaria Armenante, Maria Antonietta Crudele, \\ and Antonietta Pepe \\ Department of Science, University of Basilicata, Via Ateneo Lucano 10, 85100 Potenza, Italy \\ Correspondence should be addressed to Antonietta Pepe; antonietta.pepe@unibas.it
}

Received 5 March 2014; Accepted 7 August 2014; Published 21 August 2014

Academic Editor: Antonio Lepedda

This Conference Paper is based on a presentation given by Antonietta Pepe at "LIAC Meeting on Vascular Research 2013" held from 18 September 2013 to 21 September 2013 in Alghero, Italy.

Copyright (C) 2014 Brigida Bochicchio et al. This is an open access article distributed under the Creative Commons Attribution License, which permits unrestricted use, distribution, and reproduction in any medium, provided the original work is properly cited.

\begin{abstract}
Elastin and elastin-related peptides have great potential in the biomaterial field, because of their peculiar mechanical properties and spontaneous self-assembling behavior. Depending on their sequences and under appropriate experimental conditions, they are able to self-assemble in different fiber morphologies, including amyloid-like fibers. In this work, we will review recent data on elastin peptides derived from exon 30-coded domain of human tropoelastin. This domain has been shown to be fundamental for the correct assembly of elastin. However, the $\mathrm{N}$-terminal region forms amyloid-like fibers, while the C-terminal fragment forms elastin-like fibers. A rationale for the varied aggregation pattern has been sought in the molecular structure of the peptides. Minimal differences in the sequences, adopting alternative conformations, are shown to be responsible for the observed data.
\end{abstract}

\section{Introduction}

The development of advanced biomaterials is often inspired by the biological self-assembling modules, where simple building blocks such as amino acids, nucleic acids, and lipids are able to form complex natural systems. Peptide-based nanostructure complexes represent an important way toward the production of ordered self-assembling nanostructures with variegated possible applications $[1,2]$. The growing interest in protein-inspired bionanotechnology is based on the knowledge of different self-assembling processes involving proteins and peptides. One of the most ubiquitous selfassembly processes in nature is the hierarchical organization of protein monomers into long filaments bundles and networks of nanometric dimensions. Extracellular matrix proteins, such as elastin and collagen, are involved in different self-assembling processes, both producing well-defined fibrils and fibers with specific mechanical and supramolecular properties [3]. Among the proteins able to self-assemble, elastin and elastin-related polypeptides $[4,5]$ have peculiar characteristics with repetitive sequences of small size and complexity responsible for their self-assembling as well as for the elastic properties. Furthermore, according to their sequences, elastin peptides are able to self-assemble in two different aggregation patterns, the classical elastin-like and the amyloid-type, with mechanical properties tuned by the choice of the sequence building blocks [4]. The peculiar features of elastin-related polypeptides render them a special subject of interest, as bionanomaterial with "smart" behavior [6].

With the aim of exploring the possible use of elastin selfassembling peptides for biotechnological applications, our research group studied different fragments of the exon 30coded domain of human tropoelastin, shown to be able to form amyloid-like fibers [7]. In this paper, we will review the main results obtained on two of these fragments.

\section{Results and Discussion}

In this review, we focused our attention on the conformational and self-assembling studies of different fragments 
TABLE 1: Sequences of the studied peptides and their molecular weights.

\begin{tabular}{llc}
\hline Peptide Sequence $^{\text {a }}$ & Mw (Da) \\
\hline EX30 & GLVGAAGLGGLGVGGLGVPGVGGLG & 1961.2 \\
EX30N & GLVGAAGLGGLGVGGLG & 1324.5 \\
EX30C & LGGLGVGGLGVPGVGGLG & 1421.6 \\
\hline
\end{tabular}

atalic characters highlight the common sequence.

derived from the exon 30-coded domain of human tropoelastin (EX30), able to form amyloid-like fibers, with the aim of identifying the molecular determinants for the varied self-assembly of elastin peptides. In particular, we point our attention to the first $17 \mathrm{~N}$-terminal residues of EX30, the EX30N peptide [8], and to the $18 \mathrm{C}$-terminal residues, the EX30C peptide (Table 1) [9]. These peptides contain both the XGGZG sequences $(X, Z=V, L)$, previously suggested to be responsible for amyloid formation in elastin-related polypeptides [10]. However, the aggregation mechanism followed two different ways: EX30N peptide showed a great propensity to aggregate in water in an irreversible way, as monitored by turbidimetry experiments, suggesting the formation of amyloid-like structures [8]. The time course for aggregation is very slow at room temperature, while higher temperatures (f. ex. $50^{\circ} \mathrm{C}$ ) are able to trigger the aggregation. On the contrary, EX30C peptide is able to undergo a temperatureinduced reversible phase transition, called coacervation, observed also for elastin and tropoelastin (Figure 1). The different aggregates formed were further investigated at a nanometric scale by TEM, in order to have insights in the ultrastructure and morphology of the formed aggregates. The EX30N peptide showed typical features of amyloidlike fibers, appearing as unbranched filaments that are few nanometers in diameter and up to a micrometer or more in length (Figure 2). Conversely, EX30C peptide evidenced an elastin-like aggregation pattern, with flexible fibers and bundles of highly aligned fibers. The fibers show a twistedrope motif found in elastin and tropoelastin (Figure 3(a)). Interestingly, in Figure 3(b), the aggregates formed by EX30C peptide show also banded fibers with an elastin-like ultrastructure similar to that previously described for the coacervates of tropoelastin and $\alpha$-elastin [11].

\section{Conformational Studies}

As previously reported, the peptides were investigated by CD and NMR spectroscopy in water solution and in TFE. A summary of the CD results is presented in Table 2. The data clearly show that EX30N peptide has great tendency to populate PPII conformations in water, while EX30C peptide has more complex spectral features, assigned to a mixture of unordered and turn conformations. In TFE, a less polar solvent, EX30N and EX30C peptides show a high tendency to adopt type I and type II $\beta$-turns, respectively.

Conformational analysis of the peptides in different solutions was accomplished also by NMR spectroscopy. Secondary structure elements are derived mainly from a qualitative interpretation of NOEs and ${ }^{3} J_{\alpha \mathrm{H}-\mathrm{NH}}$ coupling

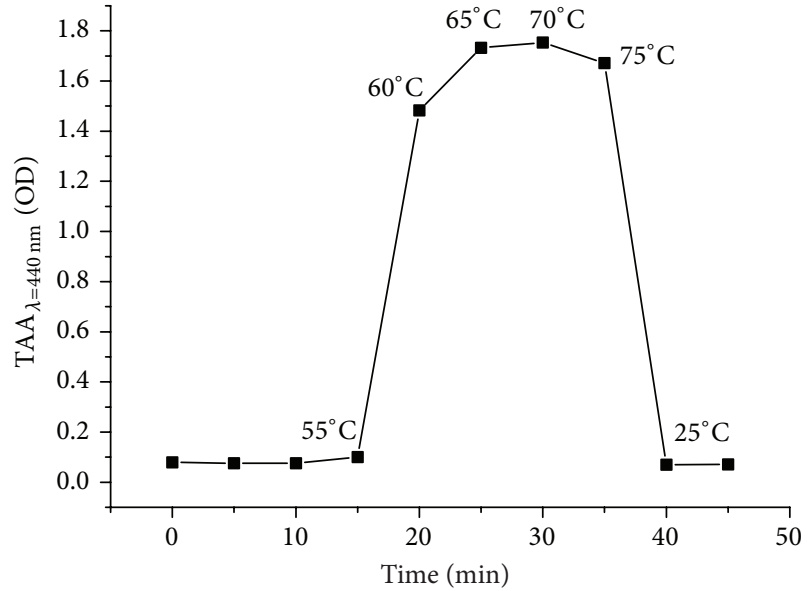

FIGURE 1: Turbidimetry on apparent absorbance (TAA) of EX30C peptide (15 $\mathrm{mM}$ in TBS) monitored during heating at the indicated temperatures.

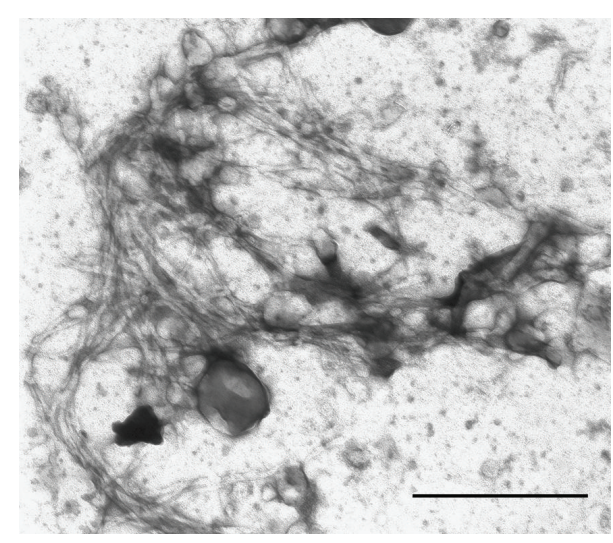

FIGURE 2: Room-temperature self-assembly of EX30N peptide in aqueous solution in amyloid-like fibers examined by TEM. Sample was negatively stained by $1 \%$ uranyl acetate solution. Bar represents $500 \mathrm{~nm}$.

constants. For both peptides, the NOESY spectra in water revealed various features consistent with the presence of PPII or unordered conformations, while turn structures were identified in TFE solution. The NOEs collected in water and in TFE were used as input data in CYANA calculations. One hundred structures were calculated, and the best 20 conformers in terms of target function values were analyzed by MOLMOL software. The EX30N peptide in aqueous solution showed an elongated structure compatible with the CD data (Figure 4(a)). No evidence of medium or long-range NOEs confirms the absence of folded structures. In TFE, a turn in the region LGGL of EX30N peptide confers a hairpin structure (Figure 4(b)) that could be responsible for the amyloid formation, whereas, for EX30C peptide, several turn structures along the polypeptide chain were identified (Figure 4(c)). The presence of several turn structures confers to the EX30C peptide a more folded structure that could account for the propensity to coacervate in more compact aggregates. 
TABLE 2: Summary of CD data recorded in water and TFE.

\begin{tabular}{llc}
\hline Peptide, experimental conditions & Spectral features (sign) & Assignments \\
\hline EX30N, water at $25^{\circ} \mathrm{C}$ & $197 \mathrm{~nm}(-) ; 217 \mathrm{~nm}(+)$ & PPII + unordered \\
EX30N, TFE at $25^{\circ} \mathrm{C}$ & $202 \mathrm{~nm}(-) ; 222 \mathrm{~nm}(-)$ & Type I $\beta$-turn + unordered \\
EX30C, water at $25^{\circ} \mathrm{C}$ & $199 \mathrm{~nm}(-) ; 217 \mathrm{~nm}(+)$ & Unordered + turns \\
EX30C, TFE at $25^{\circ} \mathrm{C}$ & $205 \mathrm{~nm}(+) ; 227 \mathrm{~nm}(-)$ & Type II $\beta$-turn + unordered \\
\hline
\end{tabular}

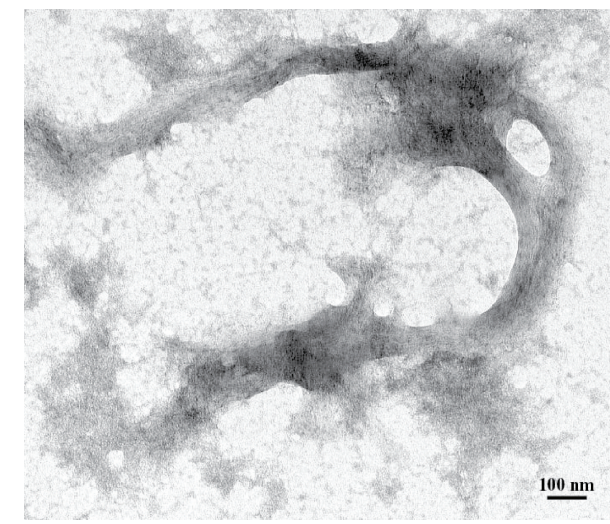

(a)

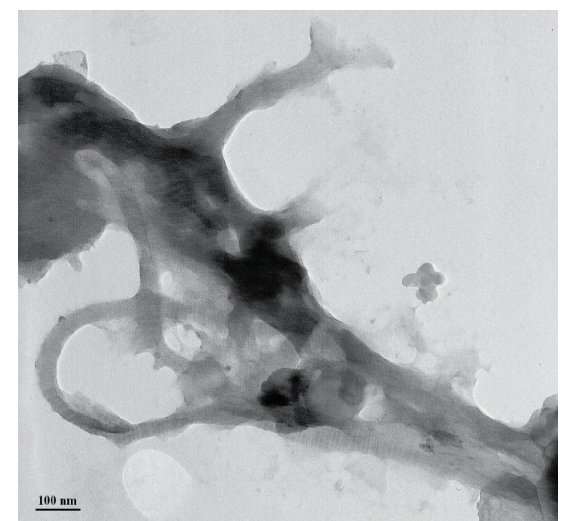

(b)

FIGURE 3: Room-temperature self-assembly of EX30C peptide in aqueous solution into elastin-like fibers examined by TEM. Sample was negatively stained by $1 \%$ uranyl acetate solution. In (a), bundles of highly aligned fibers are observed, while, in (b), the fibers evolved into banded fibers orthogonal to the fiber length.

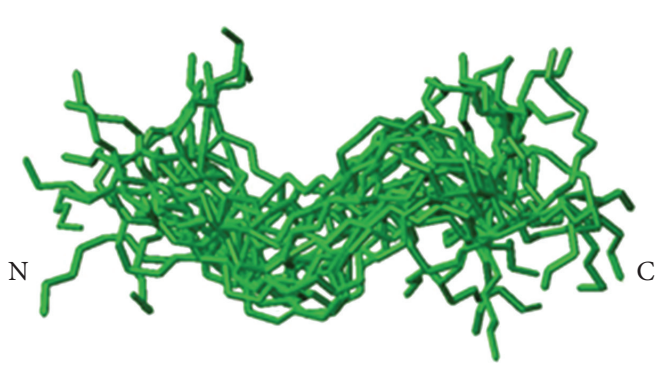

(a)

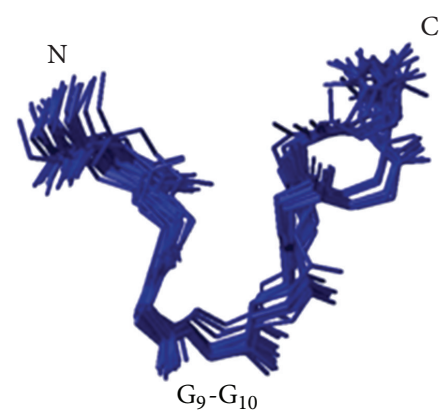

(b)

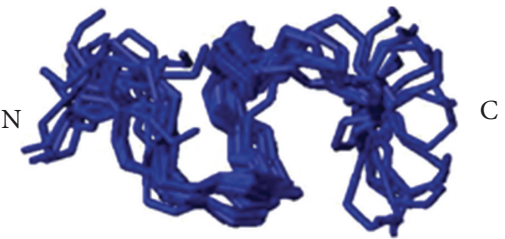

(c)

FIGURE 4: Superposition of the 20 best calculated structures of EX30N peptide in water (a) and in TFE (b) and of EX30C in TFE (c).

Similar results were observed for other elastin-related peptides. As a matter of fact, several fragments produced from the cleavages of human elastin made by some elastases were investigated for their amyloidogenic propensity [12]. Also in that case only the peptide most prone to adopt PPII conformation was shown to be able to form amyloid-like fibers. Based on these common observations, we suggest that in the case of elastin peptides the PPII conformation, a flexible structure devoid of intramolecular hydrogen bonds, coexists with $\beta$-sheet and could easily interchange into cross- $\beta$ structure. The conformational transition from PPII to cross- $\beta$ structure could be responsible for the irreversible precipitation of the soluble peptide into amyloid-like aggregates.

\section{Conclusions}

In conclusion, in this paper, we analyzed the influence of the molecular structure in defining the aggregation pattern of two closely related elastin peptides. The knowledge of the molecular determinants responsible for the alternative aggregation is fundamental for two reasons: (i) for the general comprehension of the folding of peptides and proteins and (ii) for the proper design of peptides with predictable aggregation behavior.

The possibility, under controlled experimental conditions, for some elastin-like polypeptides to form elastomeric fibers or amyloidogenic fibrils has been recently analyzed by computational methods [13]. In particular, the role of glycines 
and prolines has been studied in detail. In the choice between elastin-like and amyloidogenic peptides, these two amino acids appear to be two major sequence determinants, with proline shifting towards elastin-like aggregation. However, a not negligible role in promoting the amyloid formation has to be assigned to the first residues present in EX30 and EX30N peptides. Interestingly, the GAA motif was also found in other elastin peptides previously demonstrated to be amyloidogenic, such as EX28 [14] and EX32 [15] elastin sequences, as well as in the highly amyloidogenic AGAAAAGA peptide [16]. Therefore, it could be speculated that the GAA motif might be responsible for the major amount of PPII conformation found and for the amyloidogenic behavior of EX30N. As a consequence, when designing and producing elastinbased materials these alternative aggregation patterns have to be taken into account.

\section{Conflict of Interests}

The authors certify that no actual or potential conflict of interests in relation to this paper exists.

\section{Acknowledgments}

The authors thank A. De Stradis A. and A. Laurita for TEM micrographs. The financial support of PRIN 2010-Project 2010L (SH3 K) is gratefully acknowledged.

\section{References}

[1] S. Zhang, "Fabrication of novel biomaterials through molecular self-assembly," Nature Biotechnology, vol. 21, no. 10, pp. 11711178, 2003.

[2] F. J. Arias, V. Reboto, S. Martín, I. López, and J. C. Rodríguez-Cabello, "Tailored recombinant elastin-like polymers for advanced biomedical and nano(bio)technological applications," Biotechnology Letters, vol. 28, no. 10, pp. 687-695, 2006.

[3] J. E. Wagenseil and R. P. Mecham, "New insights into elastic fiber assembly," Birth Defects Research C Embryo Today: Reviews, vol. 81, no. 4, pp. 229-240, 2007.

[4] A. Pepe, B. Bochicchio, and A. M. Tamburro, "Supramolecular organization of elastin and elastin-related nanostructured biopolymers," Nanomedicine, vol. 2, no. 2, pp. 203-218, 2007.

[5] J. F. Almine, D. V. Bax, S. M. Mithieux et al., "Elastin-based materials," Chemical Society Reviews, vol. 39, no. 9, pp. 33713379, 2010.

[6] J. C. Rodriguez-Cabello, "Smart elastin-like polymers," Advances in Experimental Medicine and Biology, vol. 553, pp. 45-57, 2004.

[7] A. M. Tamburro, A. Pepe, B. Bochicchio, D. Quaglino, and I. P. Ronchetti, "Supramolecular amyloid-like assembly of the polypeptide sequence coded by exon 30 of human tropoelastin," The Journal of Biological Chemistry, vol. 280, no. 4, pp. 26822690, 2005.

[8] A. Pepe, M. R. Armenante, B. Bochicchio, and A. M. Tamburro, "Formation of nanostructures by self-assembly of an elastin peptide," Soft Matter, vol. 5, no. 1, pp. 104-113, 2009.

[9] A. Ostuni, B. Bochicchio, M. F. Armentano, F. Bisaccia, and A. M. Tamburro, "Molecular and supramolecular structural studies on human tropoelastin sequences," Biophysical Journal, vol. 93, no. 10, pp. 3640-3651, 2007.

[10] L. L. del Mercato, G. Maruccio, P. P. Pompa et al., "Amyloidlike fibrils in elastin-related polypeptides: structural characterization and elastic properties," Biomacromolecules, vol. 9, no. 3, pp. 796-803, 2008.

[11] D. Volpin, I. Pasquali Ronchetti, D. W. Urry, and L. Gotte, "Banded fibers in high temperature coacervates of elastin peptides," Journal of Biological Chemistry, vol. 251, no. 21, pp. 6871-6873, 1976.

[12] B. Bochicchio, A. Pepe, F. Delaunay, M. Lorusso, S. Baud, and M. Dauchez, "Amyloidogenesis of proteolytic fragments of human elastin,” RSC Advances, vol. 3, no. 32, pp. 13273-13285, 2013.

[13] S. Rauscher, S. Baud, M. Miao, F. Keeley, and R. Pomès, "Proline and glycine control protein self-organization into elastomeric or amyloid fibrils," Structure, vol. 14, no. 11, pp. 1667-1676, 2006.

[14] B. Bochicchio, A. Pepe, R. Flamia, M. Lorusso, and A. M. Tamburro, "Investigating the amyloidogenic nanostructured sequence of elastin: sequence encoded by exon 28 of human tropoelastin gene," Biomacromolecules, vol. 8, no. 11, pp. 34783486, 2007.

[15] A. M. Tamburro, M. Lorusso, N. Ibris, A. Pepe, and B. Bochicchio, "Investigating by circular dichroism some amyloidogenic elastin-derived polypeptides," Chirality, vol. 22, no. 1, pp. E56E66, 2010.

[16] M. Gasset, M. A. Baldwin, D. H. Lloyd et al., "Predicted $\alpha$-helical regions of the prion protein when synthesized as peptides form amyloid," Proceedings of the National Academy of Sciences of the United States of America, vol. 89, no. 22, pp. 10940-10944, 1992. 


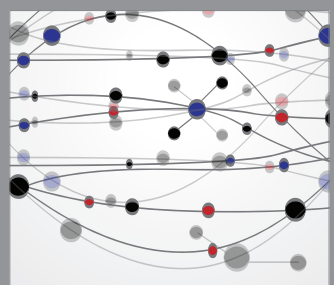

The Scientific World Journal
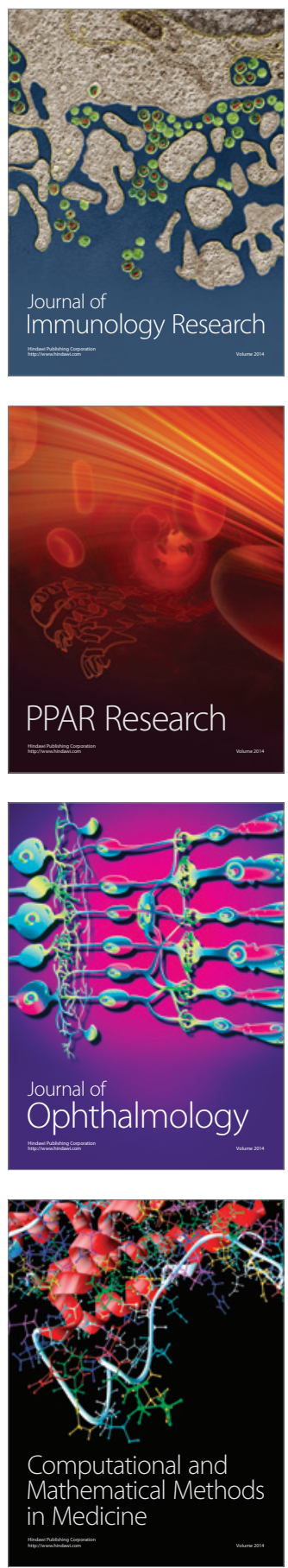

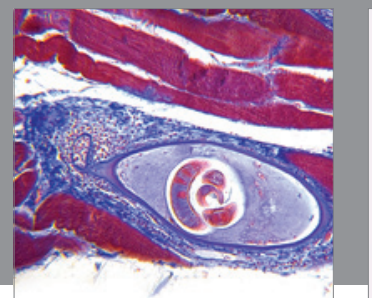

Gastroenterology

Research and Practice
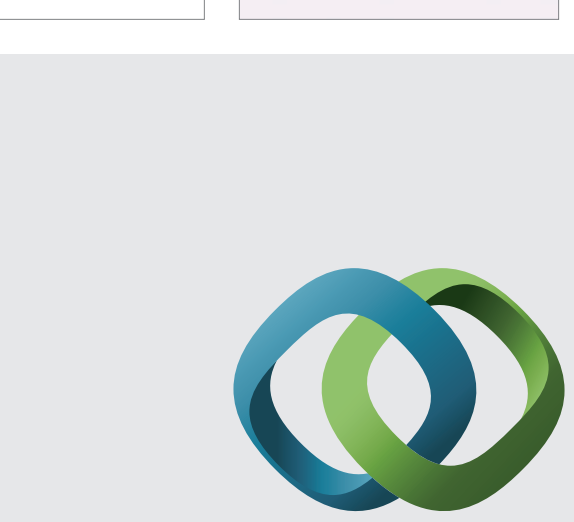

\section{Hindawi}

Submit your manuscripts at

http://www.hindawi.com
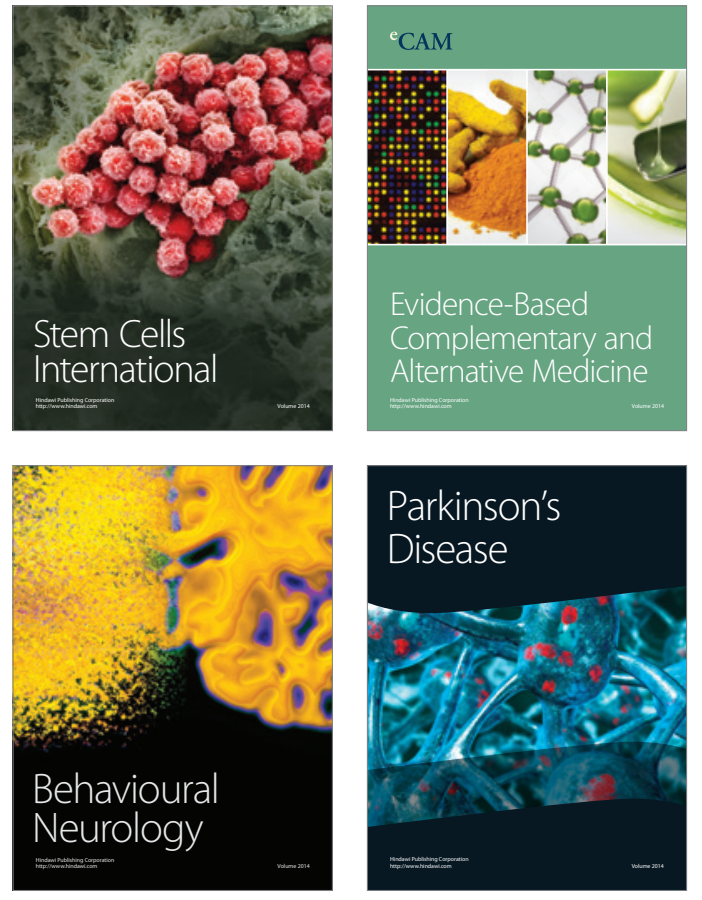
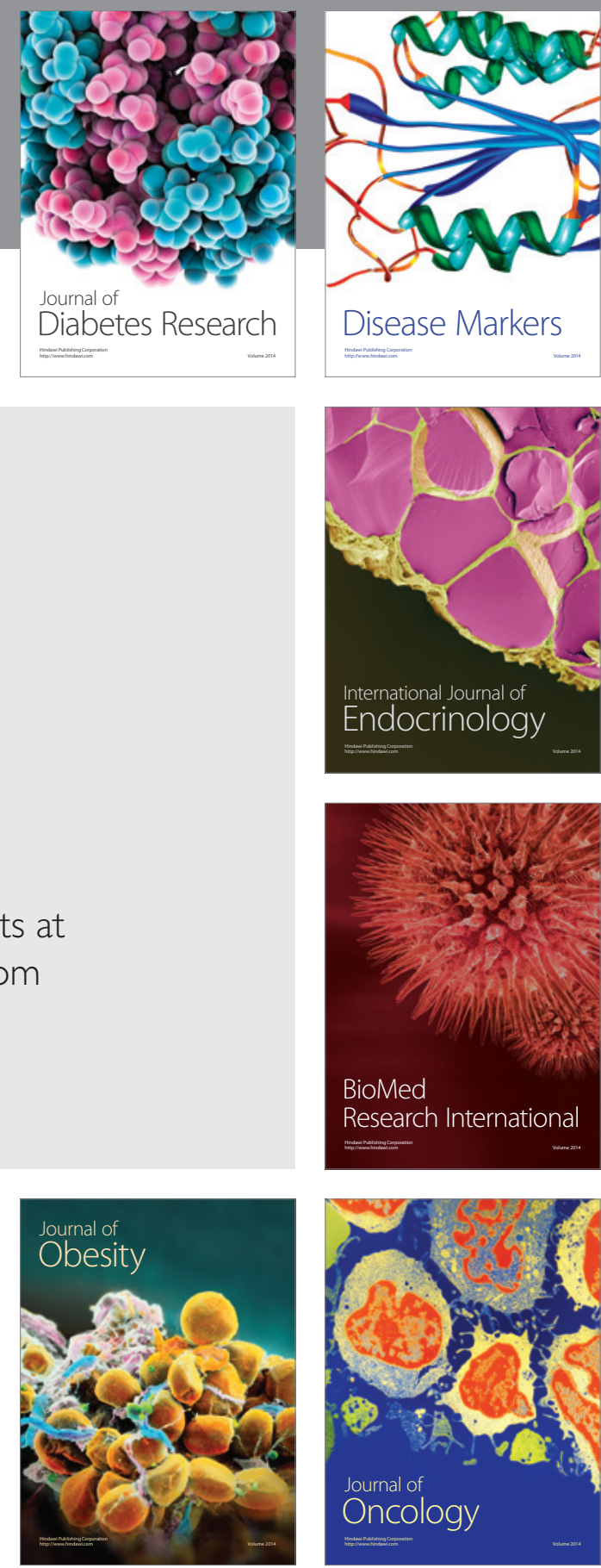

Disease Markers
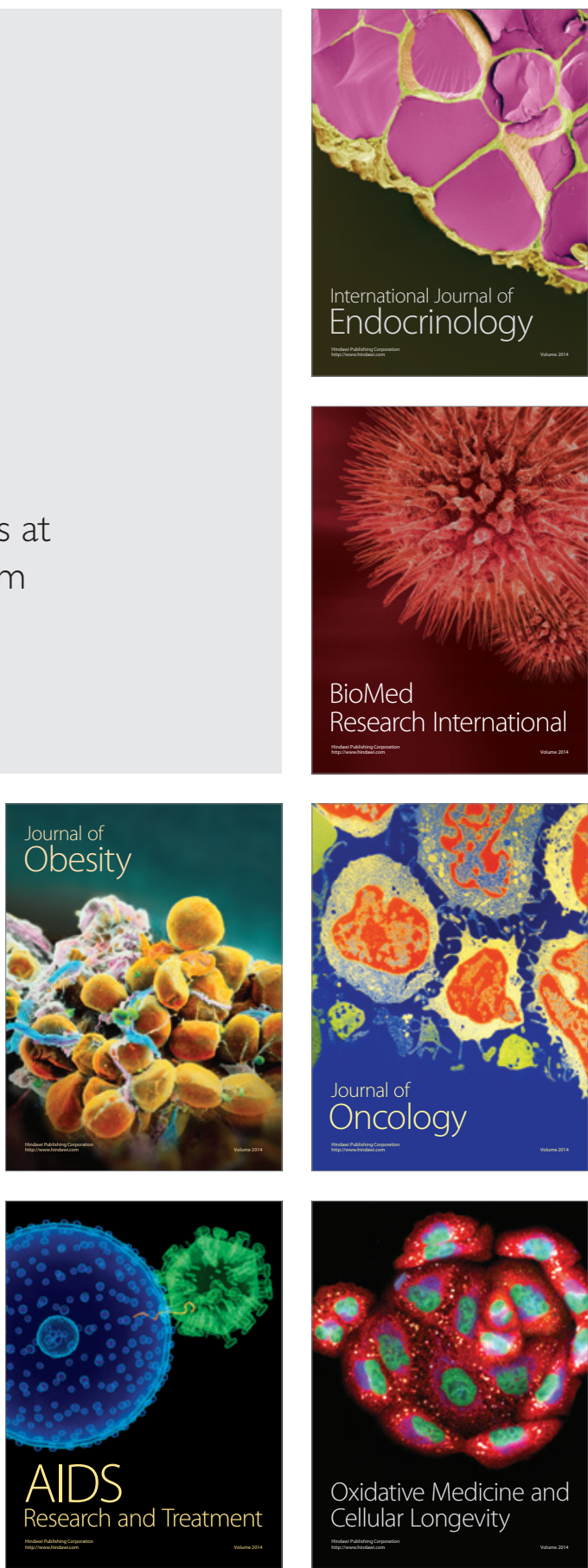